\title{
Los moluscos de la ofrenda 107 del Templo Mayor de Tenochtitlan
}

\section{The mollusks of the offering 107 of the Great Temple of Tenochtitlan}

\author{
Norma Valentín-Maldonado ${ }^{1 *}$ y Belem Zúñiga-Arellano² \\ ${ }^{1}$ Laboratorio de Arqueozoología M. C. Ticul Álvarez Solórzano, Subdirección de Laboratorios y Apoyo Académico, Instituto Nacional de Antropología \\ e Historia. Moneda 16, Centro, 06060 México D.F. \\ ${ }^{2}$ Proyecto “Técnicas de manufactura de los objetos de concha del México prehispánico”, Museo Templo Mayor, Seminario 8, Centro, 06060 México, \\ D.F. \\ *Correspondencia: nvalentinm@hotmail.mx
}

Resumen. El propósito de este trabajo es dar a conocer la identificación biológica de las especies de moluscos encontradas en la ofrenda 107, que fue depositada en la séptima etapa constructiva del Templo Mayor de Tenochtitlan (1502-1521). La identificación se realizó por medio de la bibliografía especializada, y en el caso de algunos ejemplares por comparación directa con los de la colección malacológica de referencia del Laboratorio M. en C. Ticul Álvarez Solórzano. Se identificaron 246 ejemplares de moluscos marinos; 191 pertenecen a la clase Gastropoda (78\%) y 55 a la Bivalvia (22\%). El número más frecuente de especies proviene de la provincia caribeña, siendo su hábitat principal las playas arenosas y las costas rocosas. Los desgastes y fracturas observadas en la mayoría de los esqueletos calcáreos de animales ya muertos, indican que fueron recolectados en las playas; por otro lado, el buen estado de preservación de algunos de ellos sugiere que se capturaron vivos, y en ciertos casos mediante buceo. A través de este estudio ha sido posible obtener datos, como la procedencia, el hábitat, la coloración natural y la selectividad de las diferentes especies identificadas, los cuales contribuyen al conocimiento de aspectos económicos e ideológicos de la cultura mexica.

Palabras clave: moluscos marinos, Gastropoda, Bivalvia, ofrendas, mexicas, México.

\begin{abstract}
The purpose of this work is to report the identifications of the species of mollusks found in offering 107, which was buried in the seventh stage of the construction of the Great Temple of Tenochtitlan (1502-1521). This was done by direct comparison with specimens in the malacological reference collection of the Ticul Álvarez Solorzano Laboratory and the literature. The 246 marine individuals belonging to two classes were identified; 191 belonging to the Gastropoda (78\%) and 55 to the Bivalvia (22\%). The largest number of species came from the Caribbean Province, with species from sandy beaches and rocky coasts being most common. The abrasions and fractures observed in most of the shells indicates that they were collected dead on the beaches, whereas the good state of preservation of some of them indicates that they were collected live, in some cases by diving. Through this study it has been possible to obtain data such as the provenance, habitat, natural coloration of the different species identified, and selectivity for particular ones. This has contributed to the knowledge of the economic and ideological aspects of Mexica culture.
\end{abstract}

Key words: marine mollusks, Gastropoda, Bivalvia, offering, Mexicas, Mexico.

\section{Introducción}

Desde sus inicios en 1978, el proyecto Templo Mayor planteó el estudio interdisciplinario del material recuperado en las excavaciones del templo y de los edificios aledaños que conforman el recinto sagrado de la gran Tenochtitlan. En 1991, dada la necesidad de ampliar el conocimiento producido a través de los diferentes hallazgos, se inició

Recibido: 13 noviembre 2004; aceptado: 28 marzo 2007 el Programa de Arqueología Urbana (PAU) con el fin de profundizar en el estudio del recinto sagrado de México Tenochtitlan desde la época prehispánica hasta nuestros días (Matos, 1999). El arqueólogo que coordinó dicho programa fue el profesor Eduardo Matos Moctezuma, y el responsable de la excavación el arqueólogo Álvaro Barrera Rivera.

Este programa permitió el acceso a los edificios que se intervinieron para ser restaurados, recimentados, o bien, en los que se llevaron a cabo trabajos de drenaje o 


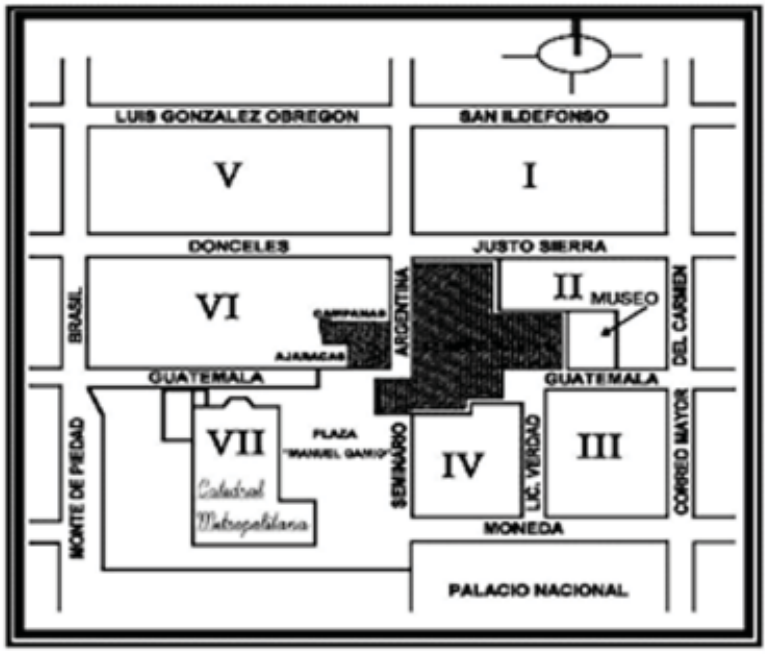

Figura 1. Área de excavación del Programa de Arqueología Urbana, Proyecto Templo Mayor (modificado de Matos Moctezuma, 1999).

de otros servicios; abarca un área de 7 manzanas e incluye el Templo Mayor (Fig. 1), la Catedral Metropolitana, la esquina noroeste del Palacio Nacional, el edificio del Apartado, la librería Porrúa, la casa con el número 33 de la calle de Justo Sierra, la Casa de las Ajaracas y la Casa de las Campanas, entre otros.

Al ser intervenidos los predios correspondientes a la casa de las Ajaracas y la de las Campanas, en la calle de Guatemala 38 y de Argentina 6 respectivamente, se localizaron 9 ofrendas; 3 de las cuales se encontraban en cistas o cajas de sillares y 6 sin contenedores, y les correspondieron los números progresivos del 99 al 107; en 8 había restos de material malacológico (Fig. 2). Las ofrendas se encontraron en el relleno de la séptima etapa constructiva del Templo Mayor de Tenochtitlan (1502-1521), frente a la escalinata del templo dedicado a Huitzilopochtli y Tlaloc.

La ofrenda 107, motivo del presente estudio, se encontró bajo un gran sillar de la plataforma de la sexta etapa constructiva, aunque corresponde a la séptima (1502-1521), sin un contenedor y del lado del templo de Tlaloc (Fig. 3).

Además de los moluscos, esta ofrenda contenía otros materiales de origen biológico, como corales, galletas de mar, erizos, percebes, 2 esqueletos de puma, restos óseos de una serpiente de cascabel, copal y 2 figuras de hule con copal e indudablemente restos de flora, que por su naturaleza no se conservaron, así como material inorgánico consistente en cuchillos de pedernal y de obsidiana y en 1500 cuentas de piedra verde. Todo esto colocado cuidadosamente, en diferentes niveles de acomodo, siguiendo un orden

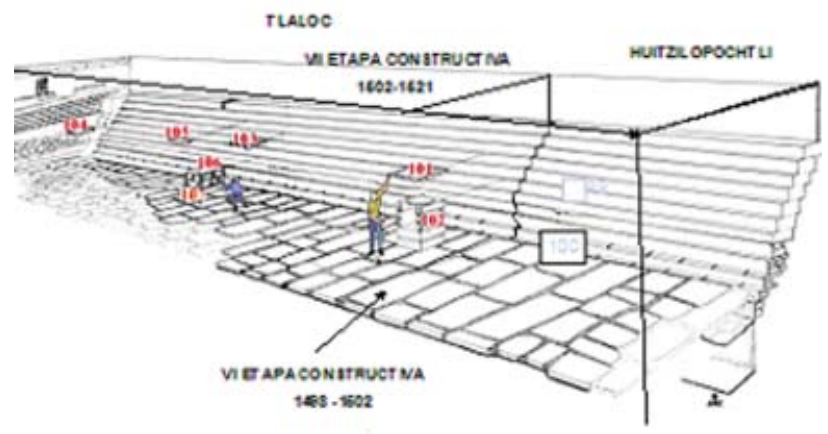

Figura 2. Ubicación de la ofrenda 107, frente a las escalinatas del Templo de Tlaloc en el relleno de la VII etapa constructiva (modificado de Álvaro Barrera).

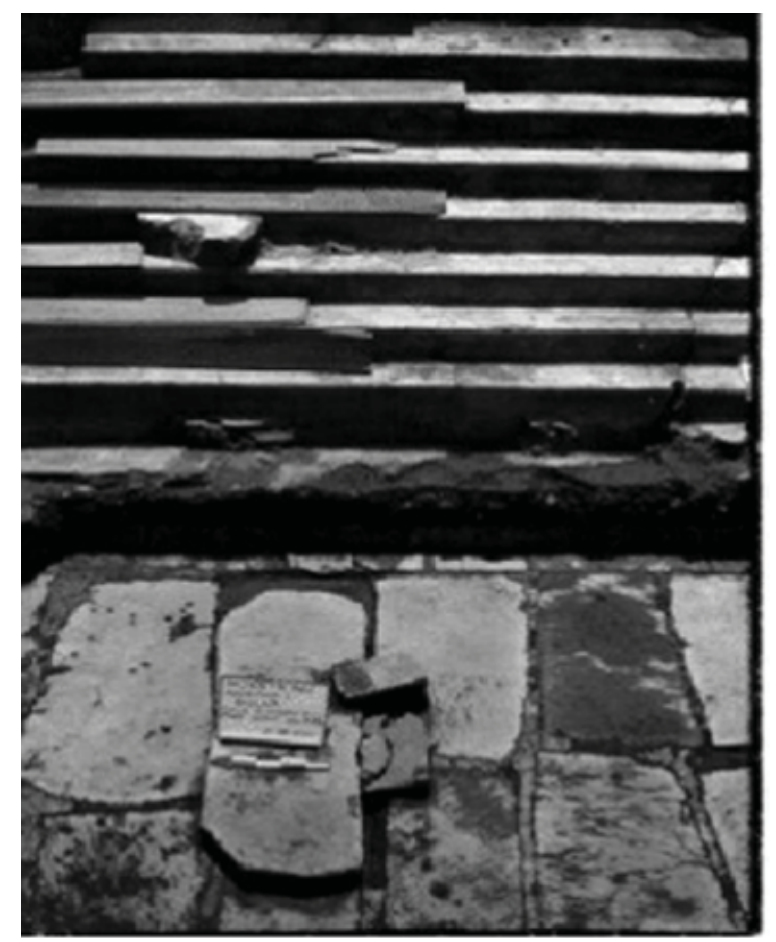

Figura 3. Ofrenda 107 tapada con el sillar de la VI etapa (15021521).

determinado, que en su conjunto comunican mensajes que se manifiestan tanto en el tipo de objetos ofrendados como en las asociaciones espaciales que se establecen entre ellos (Velázquez, 2000).

La importancia simbólica que los moluscos marinos tuvieron para el pueblo mexica se manifiesta por su presencia en las diferentes ofrendas que se hicieron para honrar los templos y edificaciones del recinto sagrado, tanto el Templo Mayor como las estructuras aledañas; en 
su interior han sido hallados un gran número de ejemplares de conchas no modificadas y más de 2000 piezas trabajadas (Velázquez, 1999) que están directamente relacionadas con el agua, el inframundo y con la petición de fertilidad a la tierra.

El significado varía dependiendo del contexto en el que se encuentre; por ejemplo, los objetos de concha al lado norte del basamento del Templo Mayor están directamente relacionados con los poderes generativos de la tierra y el agua, y los que se encuentran del lado sur parecen señalar el inframundo como el lugar en que se lleva a cabo la guerra cósmica, mediante la cual se completan los ciclos de muerte y resurrección de los astros; el Sol, Venus y las estrellas (Velázquez, 2000). El lado norte del basamento corresponde a Tlaloc, el dios pluvial en la religión mexica, y el lado sur a Huitzilopochtli, dios tutelar de la guerra. En ambos lados, los moluscos se sitúan en el fondo de las ofrendas, el inframundo; uno representa la fertilidad de la tierra y la riqueza vegetal y de alimentos; el otro, el lugar donde se llevan los procesos nocturnos de la muerte y la resurrección de los seres astrales a través de la guerra y el sacrificio (Velázquez, 2000).

La gran diversidad de especies de bivalvos y gasterópodos en el fondo de muchas de las ofrendas, junto con otros organismos marinos, como corales, esponjas, erizos o bizcochos de mar, y elementos como cascabeles de cobre y piedras verdes constituyen recreaciones del inferior de los niveles del cosmos mesoamericano (LopézLuján, 1993).

Los estudios que hasta la fecha se han llevado a cabo en el Templo Mayor sobre las identificaciones biológicas de moluscos y de los elementos de concha trabajados son los siguientes: Blanco (1978), Polaco (1982), Carramiñana (1988), López Luján y Polaco (1991), Del Olmo (1999), Velázquez (1999), Velázquez (2000), Valentín y Zúñiga (2003, 2006, 2006a en prensa), Velázquez y Zúñiga 2003), Velázquez et al. (2004).

\section{Material y métodos}

Nuestra participación se inició desde el momento mismo de la excavación, ya que se realizó una identificación previa que facilitó el registro del material faunístico, su cuantificación, y el señalamiento de la posición y acomodo de las diferentes especies dentro de la ofrenda, a fin de que los arqueólogos tuvieran todos los datos para una interpretación global. El registro consistió en dar a cada pieza un número de elemento y un número progresivo; se hicieron dibujos, se tomaron fotografías y videos y se anotaron medidas de profundidad $\mathrm{Z}$, y coordenadas ( $\mathrm{Y}$ $\mathrm{y} X$ ). Posteriormente se fueron retirando uno por uno los elementos por niveles de depósito, se etiquetaron y se guardaron por separado en bolsas y recipientes de plástico.

La mayoría de los ejemplares se encontraron en regular estado de conservación, ya que al tratarse de una ofrenda sin contenedor, el material no está protegido de los cambios del nivel freático que deslava el sedimento en el cual están acomodados, produciendo erosión en algunos; principalmente se pierde la ornamentación, la coloración y consistencia, lo que dificulta en muchos casos la identificación.

El material se limpió y el que requería ser tratado se separó para enviarlo al Departamento de Restauración del Museo del Templo Mayor. Posteriormente se trasladó a las oficinas del PAU donde se llevó a cabo el estudio.

La identificación se realizó siguiendo las características dadas por Abbott (1974) y Keen (1971) y en algunos moluscos se corroboró por comparación directa con ejemplares depositados en la colección malacológica de referencia del Laboratorio de Arqueozoología M. en C. Ticul Álvarez Solórzano, de la Subdirección de Laboratorios y Apoyo Académico, Instituto Nacional de Antropología e Historia. Para la actualización y ordenación taxonómica hasta género se siguió a Vaught (1989).

Los datos para elaborar los Cuadros 3 y 4 referentes al hábitat, distribución y localidades de las especies identificadas se obtuvieron de las revistas especializadas, libros y tesis que se citan: Emerson y Buffer (1957), Brand et al. (1960), Keen (1964, 1968, 1971), Dushane y Poorman (1967), Dushane y Sphon (1968), Abbott (1974), Toledano (1977), Vokes y Vokes (1983), Villalpando (1988), Holguín y González $(1989,1994)$, Wiley et al. (1982), Bolívar e Hidalgo (1990), Abbott y Dance (1991), Castillo (1992), Escobar (1995), Sevilla (1995).

\section{Resultados}

Se identificaron 246 moluscos de las clases Gastropoda y Bivalvia. Los primeros, con 191 ejemplares, son los más abundantes y representan el $78 \%$ del total de los moluscos estudiados; pertenecen a 9 familias, 11 géneros y 14 especies; de éstas, las más abundantes son; Fissurella rosea con 69 ejemplares, Oliva sayana y O. julieta con 24 caracoles cada una, Cittarium pica con 20 y Diodora cayenensis con 18 (Cuadro 1).

De los bivalvos se identificaron 55, que corresponden al $22 \%$ de los moluscos estudiados; pertenecen a 10 familias, 11 géneros y 11 especies; de estas últimas, las más abundantes son Chama echinata con 13 y Modiolus americanus con 12 valvas.

De la provincia caribeña proceden 16 especies y de la 
Cuadro 1. Especies identificadas en la ofrenda 107, abundancia, procedencia y posibles métodos de recolecta

\begin{tabular}{|c|c|c|c|c|}
\hline \multirow{2}{*}{$\begin{array}{c}\text { Especies } \\
\text { Phylum Mollusca }\end{array}$} & \multirow{2}{*}{$\begin{array}{l}\text { Núm. de } \\
\text { ejemplares }\end{array}$} & \multicolumn{2}{|c|}{ Procedencia } & \multirow[t]{2}{*}{ Posibles métodos de recolecta } \\
\hline & & $\begin{array}{l}\text { Provincia } \\
\text { Caribeña }\end{array}$ & $\begin{array}{l}\text { Provincia } \\
\text { Panámica }\end{array}$ & \\
\hline \multicolumn{5}{|l|}{ Clase Gastropoda } \\
\hline Familia Fissurellidae & & & & \\
\hline Diodora cayenensis & 18 & $\mathrm{X}$ & & Recolecta de playa (muertas) \\
\hline Fissurella rosea & 69 & $\mathrm{X}$ & & Recolecta de playa (muertas) \\
\hline Fissurella sp. & 1 & & & Recolecta de playa (muertas) \\
\hline Familia Trochidae & & & & \\
\hline Cittarium pica & 20 & $\mathrm{X}$ & & Vivos \\
\hline Familia Acmaeidae & & & & \\
\hline Acmaea sp. & 5 & & & Recolecta de playa (muertas) \\
\hline $\begin{array}{l}\text { Familia Strombidae } \\
\text { Strombus sp. }\end{array}$ & 1 & & & Recolecta de playa \\
\hline $\begin{array}{l}\text { Familia Crepidulidae } \\
\text { Crepidula fornicata } \\
\text { Crucibulum spinosum }\end{array}$ & $\begin{array}{l}1 \\
1\end{array}$ & $\mathrm{X}$ & $\mathrm{X}$ & $\begin{array}{l}\text { Recolecta de playa } \\
\text { Recolecta de playa }\end{array}$ \\
\hline $\begin{array}{l}\text { Familia Naticidae } \\
\text { Polinices helicoides }\end{array}$ & 1 & & $\mathrm{X}$ & Recolecta de playa \\
\hline $\begin{array}{l}\text { Familia Muricidae } \\
\text { Thais rustica }\end{array}$ & 1 & $\mathrm{X}$ & & Recolecta de playa \\
\hline $\begin{array}{l}\text { Familia Vasidae } \\
\text { Turbinella angulata }\end{array}$ & 2 & $\mathrm{X}$ & & Vivas \\
\hline Familia Olividae & & & & \\
\hline Oliva incrassata & 2 & & $\mathrm{X}$ & Playa arenosa (posiblemente vivas) \\
\hline O. julieta & 24 & & $\mathrm{X}$ & Playa arenosa (posiblemente vivas) \\
\hline O. cf. reticularis & 1 & $\mathrm{X}$ & & Playa arenosa (posiblemente vivas) \\
\hline O. sayana & 24 & $\mathrm{X}$ & & Playa arenosa (posiblemente vivas) \\
\hline O. cf. scripta & 3 & $\mathrm{X}$ & & Playa arenosa (muertas) \\
\hline O. splendidula & 8 & & $\mathrm{X}$ & Playa arenosa (posiblemente vivas) \\
\hline Oliva sp. & 7 & & & Playa arenosa (posiblemente vivas) \\
\hline Familia Conidae & & & & \\
\hline $\begin{array}{l}\text { Conus sp. } \\
\text { Caracol no identificado }\end{array}$ & $\begin{array}{l}1 \\
1\end{array}$ & & & Playa arenosa (muertos) \\
\hline Clase Bivalvia & & & & \\
\hline Familia Arcidae & & & & \\
\hline Arca imbricata & 4 & $\mathrm{X}$ & & Playa arenosa (muertas) \\
\hline Anadara chemnitzii & 7 & $\mathrm{X}$ & & Playa arenosa (muertas) \\
\hline $\begin{array}{l}\text { Familia Mytilidae } \\
\text { Modiolus americanus }\end{array}$ & 12 & $\mathrm{X}$ & $\mathrm{X}$ & Playa rocosa (vivas) \\
\hline
\end{tabular}


Familia Pteriidae

Pinctada mazatlanica

Familia Pectinidae

Aequipecten muscosus

Familia Spondylidae

Spondylus calcifer

S. princeps

Spondylus sp.

Familia Carditidae

Carditamera floridana

Familia Chamidae

Chama echinata

Chama sp.

Familia Mactridae

Rangia flexuosa

Familia Donacidae

Donax sp.

Familia Veneridae

Dosinia discus

Concha no identificada
1

1

X

$\begin{array}{ll}\text { X } & \text { Buceo } \\ \text { X } & \text { Buceo } \\ & \text { Buceo }\end{array}$

X

13

4

5

X

1

$\mathrm{X}$

Buceo

Buceo

X Buceo
Playa rocosa (posiblemente viva)

Playa arenosa (muertas)

Playa arenosa (muertas)

Playa arenosa (muertas)

Playa arenosa (muertas)

Playa arenosa (muertas)

Playa arenosa (muertas)

$\begin{array}{llll}\text { Total de especies por } & 246 & 16 & 10\end{array}$

panámica 10. El hábitat más explotado fue el de las playas arenosas en la zona mesolitoral. Varios de los ejemplares arqueológicos presentan erosión, abrasión y decoloración. Algunos se encontraron en buen estado de conservación, lo que sugiere que se recolectaron vivos (Cuadros 1 y 2).

\section{Discusión}

E1 78\% de los moluscos en la ofrenda son gasterópodos. Fissurella rosea, con 69 ejemplares, es la especie más abundante. El género Oliva, también con 69 ejemplares, tuvo 2 especies, $O$. julieta y $O$. sayana, que provienen de las provincias panámica y caribeña, respectivamente, con 24 ejemplares cada una.

La mayoría de las especies identificadas viven en aguas poco profundas cerca de la línea de costa, lo que facilita su obtención. Los desgastes y fracturas observadas en muchos de los esqueletos calcáreos indican que fueron recolectados en las playas; por ejemplo, la gran cantidad de F. rosea (69) posiblemente se deba a la facilidad que representa recolectarlos en las playas arenosas, ya que comúnmente una vez muerto el molusco se separa del sustrato rocoso donde habita y es arrastrado por la corriente hacia las playas arenosas, y lo mismo puede decirse de otras especies que se han encontrado, como Diodora cayenensis. Por el contrario, el buen estado de preservación de algunos esqueletos indica que los moluscos fueron recolectados vivos en las playas arenosas y rocosas de fácil acceso; por ejemplo, 2 ejemplares de Turbinella angulata que aún presentaban fragmentos del periostracum; algunos caracoles de Oliva sayana, O. julieta, O. spendidula, O. scripta y O. reticularis en buen estado, conservan una coloración tenue; inclusive, otros de Oliva, aunque fragmentados, se puede observar que estuvieron trabajados en forma de pendientes y que el deterioro se originó después de que se depositó en la ofrenda.

En esta oblación, Cittarium pica está representada con 20 ejemplares en buen estado que posiblemente se recolectaron vivos. Es interesante la colocación de estos caracoles que enmarcaban claramente los elementos marinos y las cuentas de piedra verde que se encontraron en el fondo de la ofrenda formando un sartal (Fig. 4). 
Cuadro 2. Hábitat de las especies identificadas registradas según la literatura

\begin{tabular}{|c|c|c|c|}
\hline Especies & $\begin{array}{l}\text { Meso- } \\
\text { litoral }\end{array}$ & $\begin{array}{l}\text { Sub- } \\
\text { litoral }\end{array}$ & \\
\hline Diodora cayenensis & $\mathrm{X}$ & & $\begin{array}{l}\text { Sustrato rocoso, manglares, arrecifes coralinos (Vokes y } \\
\text { Vokes, 1983). }\end{array}$ \\
\hline Fissurella rosea & $\mathrm{X}$ & & Sobre las rocas (Abbott y Dance, 1991) \\
\hline Cittarium pica & $\mathrm{X}$ & & Arrecifes coralinos (Vokes y Vokes, 1983) \\
\hline Crepidula fornicata & $\mathrm{X}$ & & Adheridos a las rocas o a otras conchas (Abbott, 1974) \\
\hline Crucibulum spinosum & $\mathrm{X}$ & & $\begin{array}{l}\text { Fondo arenoso a } 33 \text { metros de profundidad (Emerson, } \\
\text { 1957), recolectados mediante dragado de } 1-25 \text { metros de } \\
\text { profundidad (Keen, 1964; Strong et al., 1933) }\end{array}$ \\
\hline Polinices helicoides & $\mathrm{X}$ & & $($ Keen, 1971) \\
\hline Thais rustica & $\mathrm{X}$ & & $\begin{array}{l}\text { Sustrato rocoso, arrecifes coralinos (Vokes y Vokes, } \\
\text { 1983) }\end{array}$ \\
\hline Turbinella angulata & $\mathrm{X}$ & & $\begin{array}{l}\text { Fondos arenosos, manglar, arrecifes coralinos (Vokes y } \\
\text { Vokes, 1983) }\end{array}$ \\
\hline Oliva incrassata & $\mathrm{X}$ & & Fondos arenosos (Keen, 1971) \\
\hline O. julieta & $\mathrm{X}$ & & Fondos arenosos (Keen, 1971) \\
\hline O. reticularis & & $\mathrm{X}$ & $\begin{array}{l}\text { Fondos arenosos, arrecifes coralinos, manglares (Vokes } \\
\text { y Vokes, 1983) }\end{array}$ \\
\hline O. sayana & $\mathrm{X}$ & & Manglares, fondos arenosos (Vokes y Vokes, 1983) \\
\hline O. scripta & $\mathrm{X}$ & & $\begin{array}{l}\text { Fondos arenosos cerca de la costa (Vokes y Vokes, } \\
\text { 1983) }\end{array}$ \\
\hline O. spendidula & $\mathrm{X}$ & & Fondos arenosos (Keen, 1971) \\
\hline Arca imbricata & $\mathrm{X}$ & & $\begin{array}{l}\text { Adherida a las rocas en aguas someras, Manglares, } \\
\text { fondos arenosos, arrecifes coralinos (Vokes y Vokes, } \\
\text { 1983) }\end{array}$ \\
\hline Anadara chemnitzii & $\mathrm{X}$ & & \\
\hline Modiolus americanus & $\mathrm{X}$ & & $\begin{array}{l}1 \text { a } 6 \text { metros de profundidad (Abbott, 1974; Keen, 1971), } \\
\text { adherido a las rocas (observación personal) }\end{array}$ \\
\hline Pinctada mazatlanica & $\mathrm{X}$ & & $\begin{array}{l}\text { Adheridas a las rocas, playa arenosa, playa rocosa y } \\
\text { posiblemente en arrecife coralino (Emerson, 1957), en la } \\
\text { zona intermareal (Keen, 1964) }\end{array}$ \\
\hline Aequipecten muscosus & & & $\begin{array}{l}\text { Manglares, arrecifes coralinos, fondos arenosos (Vokes y } \\
\text { Vokes, 1983) }\end{array}$ \\
\hline Spondylus calcifer & & $\mathrm{X}$ & $\begin{array}{l}\text { Sustratos rocosos (Abbott, 1974; Keen, 1971), playa de } \\
\text { arena gruesa (Emerson, 1957) }\end{array}$ \\
\hline S. princeps & & $\mathrm{X}$ & $\begin{array}{l}\text { Playa arenosa, playa rocosa y posiblemente en arrecife } \\
\text { coralino (Emerson, 1957); también se ha obtenido por } \\
\text { dragado de } 22 \text { a } 80 \text { metros de profundidad (Keen, 1964) }\end{array}$ \\
\hline Carditamera floridana & $\mathrm{X}$ & & $\begin{array}{l}\text { Manglares, fondos arenosos, costa rocosa (Vokes y } \\
\text { Vokes, 1983) }\end{array}$ \\
\hline Chama echinata & $\mathrm{X}$ & & Sustratos duros (Keen, 1971) \\
\hline Rangia flexuosa & $\mathrm{X}$ & & Manglares (Abbott, 1974) \\
\hline Dosinia discus & $\mathrm{X}$ & & Fondos arenosos (Abbott, 1974) \\
\hline
\end{tabular}

Zona mesolitoral (intermareal o intertidal): se encuentra entre las líneas de marea más alta y la más baja.

Zona sublitoral o infralitoral: se extiende más allá del límite de la marea más baja y continúa hasta el término de la plataforma continental, es típicamente marina y muy rica en vida. 


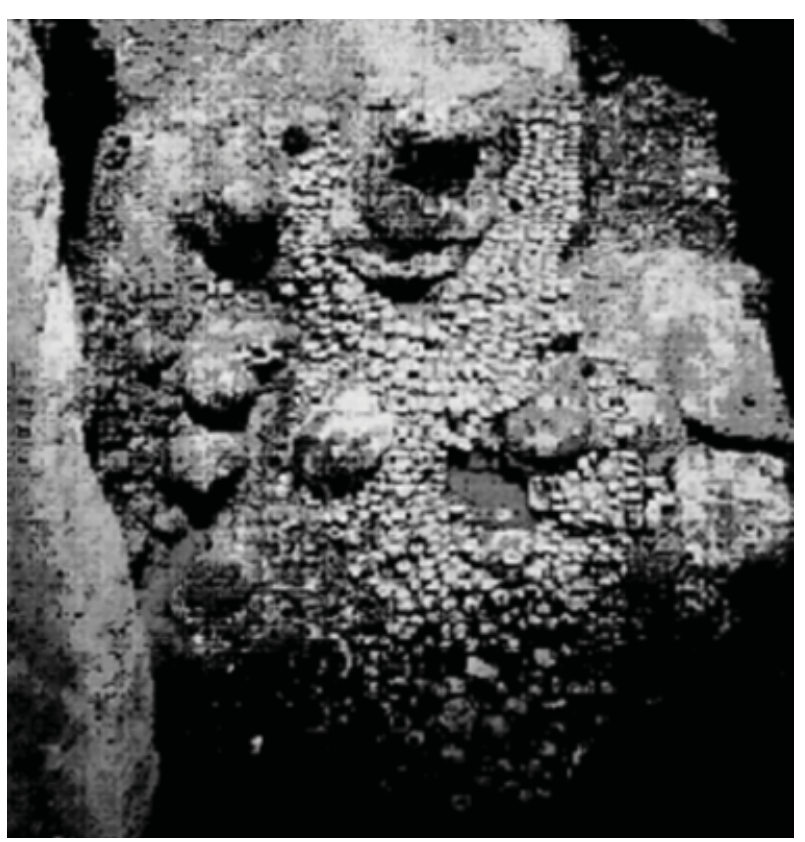

Figura 4. Fondo de la ofrenda 107.

Tomando en cuenta su hábitat, posiblemente algunas especies en buen estado de conservación se obtuvieron mediante buceo, como Spondylus calcifer, de la cual se identificaron las 2 valvas de un mismo ejemplar de gran tamaño, y 13 ejemplares de Chama echinata.

Es interesante observar que la mayoría de los bivalvos que presentan coloración roja, naranja o morada proceden de la provincia panámica, lo que, por venir de lugares más lejanos, hace más valiosa su presencia en la ofrenda; tal es el caso de C. echinata, S. princeps, S. calcifer y Modiolus americanus, esta última en ambos litorales (Abbott, 1974; Keen, 1971). Estas especies, aunque se presentan en número menor, tienen importancia simbólica y jerárquica para el pueblo mexica y en general para los pueblos prehispánicos. Al conjunto de estas especies y otras de colores semejantes se les conocía como tapachtli (coral, concha o venera de color rojo encendido) (Temple y Velázquez, 2003), por lo que concluimos que muy posiblemente eran traídas al Templo Mayor sobre pedido.

Las especies presentes en la ofrenda que consideramos eran encargadas por su abundancia y por el trabajo que se les hizo, son los caracoles Oliva sayana, O. julieta, O. spendidula, O. incrassata, O. reticularis, O. scripta, Turbinella angulata, Citarium pica, y los bivalvos Pinctada mazatlanica, Spondylus calcifer, S. princeps y C. echinata. Vale la pena aclarar que aunque algunas de las especies están en menor cantidad, en esta ofrenda ocupan un lugar especial. Así mismo, en otras ofrendas del templo, $P$. mazatlanica y T. angulata se trabajaron transformándolas en ornamentos preciados, como por ejemplo el anahuatl y el ehecacózcatl, por mencionar algunos (Velázquez, 1999; Velázquez, Mendoza y Valentín, 2004; Velázquez y Melgar, 2006).

Según la bibliografía consultada, si bien muchas de las especies tienen una distribución muy amplia tanto en la provincia caribeña como en la panámica, es posible proponer con cierta certeza la procedencia de algunas de las especies identificadas. Para la provincia caribeña sugerimos el estado de Campeche; para la panámica, la zona de Oaxaca a Michoacán (Cuadros 3 y 4).

La posible procedencia de los moluscos del Atlántico es el estado de Campeche, lo que coincide con las fuentes históricas que mencionan un importante puerto comercial que se cree estaba entre Tabasco y Campeche, llamado Xicalango, de donde llegaban los moluscos a la gran Tenochtitlan (Velázquez y Zuñiga, 2003).

En el caso de la posible procedencia de los caracoles de la provincia panámica, las especies que nos pueden indicar o proponer algo son Polinices helicoides y O. julieta; para esta última Sterba (2004) menciona que se encuentra desde el sur de México hasta Perú y Keen (1971) la registra para Matanchen, Nayarit, México; se puede observar que la mayor concentración de especies registradas se encuentra en Michoacán (Cuadro 4). Para este caso podemos aludir a las fuentes históricas que mencionan la provincia de Cihuatlan, que se encontraba entre Acapulco y Colima (Velázquez, 1999); sin embargo, considerando la distribución del bizcocho de mar Clypeaster spaciosus (identificada por F. Solís Marín) encontrada en la misma ofrenda, posiblemente los moluscos procedan de regiones más norteñas.

Se sabe que los diversos factores que inciden en las poblaciones pueden variar dependiendo de la hora de recolecta, de la temporada del año, del colector, etc.; cuando se haga el análisis de todas las especies identificadas tendremos más datos y elementos que ayudarán a obtener un panorama global para corroborar lo que aquí proponemos.

Con la información presentada de las especies de moluscos de la ofrenda 107 y de las diferentes ofrendas que han sido estudiadas del Templo Mayor de Tenochtitlan, así como los datos sobre su procedencia, hábitat, colorido natural y selectividad de las diferentes especies, se ha ampliado el conocimiento sobre los aspectos económicos e ideológicos de la cultura del mexica.

\section{Literatura citada}

Abbott, R. T. 1974. American sea shells, Van Nostrand, 
Cuadro 3. Especies de la provincia caribeña

\begin{tabular}{lcccccc}
\hline \multicolumn{1}{c}{ Estados/Especies } & Tamaulipas & Veracruz & Tabasco & Campeche & Yucatán & Quintana Roo \\
\hline Diodora cayenensis & & $\mathrm{X}$ & & $\mathrm{X}$ & $\mathrm{X}$ & $\mathrm{X}$ \\
Fissurella rosea & & $\mathrm{X}$ & & $\mathrm{X}$ & & $\mathrm{X}$ \\
Cittarium pica & & $\mathrm{X}$ & $\mathrm{X}$ & $\mathrm{X}$ \\
Crepidula fornicata & $\mathrm{X}$ & $\mathrm{X}$ & & $\mathrm{X}$ & $\mathrm{X}$ & $\mathrm{X}$ \\
Thais rustica & & $\mathrm{X}$ & & & $\mathrm{X}$ & $\mathrm{X}$ \\
Turbinella angulata & $\mathrm{X}$ & $\mathrm{X}$ & & $\mathrm{X}$ & $\mathrm{X}$ & $\mathrm{X}$ \\
Oliva reticularis & $\mathrm{X}$ & $\mathrm{X}$ & & $\mathrm{X}$ & $\mathrm{X}$ & $\mathrm{X}$ \\
O. sayana & & & $\mathrm{X}$ & $\mathrm{X}$ & $\mathrm{X}$ \\
O. scripta & $\mathrm{X}$ & $\mathrm{X}$ & & $\mathrm{X}$ & $\mathrm{X}$ & $\mathrm{X}$ \\
Arca imbricata & $\mathrm{X}$ & $\mathrm{X}$ & $\mathrm{X}$ & $\mathrm{X}$ & & $\mathrm{X}$ \\
Anadara chemnitzii & $\mathrm{X}$ & $\mathrm{X}$ & & & & \\
Modiolus americanus & $\mathrm{X}$ & $\mathrm{X}$ & & $\mathrm{X}$ & $\mathrm{X}$ & $\mathrm{X}$ \\
Aequipecten muscosus & $\mathrm{X}$ & $\mathrm{X}$ & $\mathrm{X}$ & $\mathrm{X}$ & & $\mathrm{X}$ \\
Carditamera floridana & $\mathrm{X}$ & $\mathrm{X}$ & $\mathrm{X}$ & & $\mathrm{X}$ & $\mathrm{X}$ \\
Rangia flexuosa & & & & & & \\
Dosinia discus & & & & & \\
\hline
\end{tabular}

Cuadro 4 . Especies de moluscos de la provincia panámica

\begin{tabular}{|c|c|c|c|c|c|c|c|c|c|c|}
\hline Especies & B.C. Sur & Son. & Sin. & Nay. & Jal. & Col. & Mich. & Gro. & Oax. & Chis. \\
\hline Crucibulum spinosum & $\mathrm{X}$ & $\mathrm{X}$ & & $\mathrm{X}$ & $\mathrm{X}$ & $\mathrm{X}$ & $\mathrm{X}$ & $\mathrm{X}$ & $\mathrm{X}$ & \\
\hline Polinices helicoides & & & & & & & $\mathrm{X}$ & $X$ & & \\
\hline Oliva incrassata & $\mathrm{X}$ & $\mathrm{X}$ & & & & & & & $\mathrm{X}$ & $\mathrm{X}$ \\
\hline O. julieta & & & & $\mathrm{X}$ & & & $\mathrm{X}$ & & & $\mathrm{X}$ \\
\hline O. spendidula & & & & $\mathrm{X}$ & & & $\mathrm{X}$ & & & \\
\hline Pinctada mazatlanica & $\mathrm{X}$ & $\mathrm{X}$ & $\mathrm{X}$ & & $X$ & $\mathrm{X}$ & $\mathrm{X}$ & $\mathrm{X}$ & $\mathrm{X}$ & \\
\hline Spondylus calcifer & $\mathrm{X}$ & $\mathrm{X}$ & & $\mathrm{X}$ & $\mathrm{X}$ & & $\mathrm{X}$ & & $\mathrm{X}$ & \\
\hline S. princeps & $\mathrm{X}$ & $\mathrm{X}$ & & & & & & & & \\
\hline Chama echinata & & & & & & & & & $\mathrm{X}$ & \\
\hline
\end{tabular}

New York. 541 p.

Abbott, R. T. y S. P. Dance. 1991. Compendium of Seashells. Charles Letts, London. 411 p.

Blanco Padilla, A. 1978. Análisis de los materiales biológicos en las ofrendas a Coyolxauhqui. Boletín INAH, $3^{a}$ época 24:31-38.

Bolívar de Carranza, A.M. y E. Hidalgo Escalante. 1990. Lista de moluscos gastrópodos y pelecypodos del
Golfo de México y el Caribe. Anales de la Escuela Nacional de Ciencias Biológicas 3:53-72.

Brand, D. D., C. E. Singletary, B. L. Tuner, F. M. Bullard, J. A. Peters, R. W. Storer y J. Corona Nuñez. 1960. Coalcoman and Motines de Oro, an exdistrict of Michoacán, Mexico. The Institute of Latin American Studies, University of Texas, Austin. 180 p.

Carramiñana, A. E. 1988. Informe preliminar sobre la 
ofrenda zoológica dedicada a Coyolxauqui, Dirección de Monumentos Prehispánicos, Arqueología, INAH 3:225-250.

Castillo, F. M. 1992. Sistemática, distribución, abundancia y variación estacional de los moluscos gasterópodos de la plataforma continental de Jalisco, México. Tesis, Facultad de Ciencias, Universidad Nacional Autónoma de México, México, D.F. 200 p.

Del Olmo Frese, L. 1999. Análisis de la ofrenda 98 del Templo Mayor de Tenochtitlan, INAH, Colección Científica 384, Instituto Nacional de Antropología e Historia, México, D.F. 233 p.

Dushane, H. y R. Poorman. 1967. A checklist of mollusks for Guaymas, Sonora, Mexico. The Veliger 9:413440.

Dushane, H. y G. G. Sphon. 1968. A checklist of intertidal mollusks for Bahía Willard and the southwestern portion of Bahía San Luis Gonzaga State of Baja California. The Veliger 10:233-246.

Emerson, W. K. y E. L. Buffer. 1957. Recent mollusks of the 1940 "E.W. Scripps" Cruise to the Gulf of California. American Museum Novitates 1825:1-57.

Escobar de la Llata, F. F. 1995. Moluscos de la plataforma y talud continentales de la península de Yucatán: Distribución y hábitos. Tesis maestría Facultad de Ciencias, División de Estudios de Postgrado, Universidad Nacional Autónoma de México, México, D.F. 180 p.

Holguín Quiñones, O. y A. González. 1989. Moluscos de la franja costera del estado de Oaxaca, México, Atlas CICIMAR 7, Centro Interdisciplinario de Ciencias del Mar, Instituto Politécnico Nacional, México, D.F. $221 \mathrm{p}$.

Holguín Quiñones, O. y A. González. 1994. Moluscos de la franja costera de Michoacán, Colima y Jalisco, México. Escuela Nacional de Ciencias Biológicas, Instituto Politécnico Nacional, México, D.F. 133 p.

Keen, A. M. 1964. A quantitative analysis of molluscan collections from Isla Espíritu Santo, Baja California, Mexico. Proceedings of the California Academy of Sciences. Fourth Series, 30:175-206.

Keen, A. M. 1968. West American Mollusk Types at the British Museum (Natural History) IV. Carpenter's Mazatlan Collection. The Veliger 10:389-439.

Keen A. M.1971. Sea shells of tropical West America, Stanford University Press, Stanford. 1064 p.

López Luján, L. y O. J. Polaco. 1991. La fauna de la ofrenda H del Templo Mayor. In La fauna en el Templo Mayor, O. Polaco (coord.). Instituto Nacional de Antropología e Historia, México, D.F. 149-169 p.

López Luján, L. 1993. Las ofrendas del Templo Mayor de Tenochtitlan. Instituto Nacional de Antropología e
Historia, México, D.F. 432 p.

Matos Moctezuma, E. 1999. El Programa de Arqueología Urbana. In Excavaciones en la Catedral y el Sagrario Metropolitanos, Programa Arqueología Urbana, E. Matos Moctezuma. (coord.). Colección Obra Diversa, Instituto Nacional de Antropología e Historia, México, D.F. p. 9-14 .

Polaco, O. J. 1982. Los invertebrados de la ofrenda 7 del Templo Mayor. In El Templo Mayor: excavaciones y estudios, E. Matos Moctezuma (coord.). Instituto Nacional de Antropología e Historia, México, D.F. p. 143-150.

Sevilla Hernández, M. L. 1995. Moluscos de la franja costera de Chiapas, México. Instituto Politécnico Nacional, México, D.F. 152 p.

Sterba, G. H. W. 2004. Olividae: a collectors guide. Conchbooks, Gent (Bélgica). 172 p.

Strong, A. M., G. D. Hanna y L. G. Hertlein. 1933. The Templeton Crocker Expedition of the California Academy of Sciences, 1932. Marine Mollusca from Acapulco, Mexico with notes on other species. Proceedings of the California Academy of Sciences, Fourth Series 21:117-130.

Temple Sánchez-Gavito J. J. y A. Velázquez Castro. 2003, El tapachtli entre los antiguos nahuas. In Estudios etnobiológicos. Pasado y presente de México, A. Montúfar López (coord.). Obra Varia. Instituto Nacional de Antropología e Historia, México, D.F. p. 15-23.

Toledano Granados, A. G. 1977. Estudio preliminar de la fauna malacológica de la laguna de Agiabampo, Sonora-Sinaloa, México. Tesis, Facultad de Ciencias. Universidad Nacional Autónoma de México, México, D.F. 155 p.

Valentín Maldonado, N. y B. Zúñiga Arellano. 2003. La fauna de la ofrenda 103 del Templo Mayor de Tenochtitlan. In Estudios etnobiológicos. Pasado y presente de México, A. Montúfar López (coord.) Obra Varia. Instituto Nacional Antropología e Historia, México, D.F. p. 63-68.

Valentín Maldonado, N. y B. Zúñiga Arellano. 2006. La fauna en la ofrenda 102, Templo Mayor de Tenochtitlan. In Arqueología e Historia del Centro de México: Homenaje a Eduardo Matos Moctezuma. Instituto Nacional de Antropología e Historia, México, D.F. p. 507-524.

Valentín Maldonado, N. y B. Zúñiga Arellano, 2006a. En prensa. La identificación de los restos fáusticos de las ofrendas 99 y 100 del Templo Mayor de Tenochtitlan, vol. 3, Programa de Arqueología Urbana, Templo Mayor. Instituto Nacional Antropología e Historia, México, D.F. 
Vaught, C. K. 1989. Classification of the living Mollusca, T. Abbott and K. J. Boss (eds.). American Malacologist, Melbourne, Florida. 189 p.

Velázquez Castro, A. 1999. Tipología de los objetos de concha del Templo Mayor de Tenochtitlan. Colección Científica 392. Instituto Nacional de Antropología e Historia, México, D.F. 134 p.

Velázquez Castro, A. 2000. El simbolismo de los objetos de concha encontrados en las ofrendas del Templo Mayor de Tenochtitlan. Colección Científica 403. Instituto Nacional de Antropología e Historia, México, D.F. 321 p.

Velázquez Castro, A. y B. Zúñiga Arellano. 2003. Los pendientes de caracoles del género Oliva de las ofrendas del Templo Mayor de Tenochtitlan. In Estudios etnobiológicos. Pasado y presente de México. A. Montúfar López (coord.). Obra Varia. Instituto Nacional de Antropología e Historia, México, D.F. p. 27-38.

Velázquez Castro, A., B. Zúñiga Arellano y N. Valentín Maldonado. 2004. Ofrendas de concha: tesoros de fertilidad, CONACULTA/ INAH / Museo del Templo Mayor/ Asociación de Amigos del Templo Mayor, México, D.F. 63 p.

Velázquez Castro, A., D. Mendoza Anaya y N. Valentín
Maldonado. 2004. Los anahuatl de concha del Templo Mayor de Tenochtitlan: su valor visto a través de sus técnicas de manufactura. In Las ciencias materiales y su impacto en la arqueología, D. Mendoza Anaya, E. L. Brito Benítez y J. Arenas Alatorre (eds.). Academia Mexicana de Ciencias Materiales, Innovación Editorial Lagares de México, México, D.F. p. 129-140.

Velázquez Castro, A. y E. R. Melgar Tísoc. 2006. Los ehecacózcatl de concha del Templo Mayor de Tenochtitlan. In Arqueología e historia del centro de México: Homenaje a Eduardo Matos Moctezuma. Instituto Nacional de Antropología e Historia, México, D.F. p. 525-537.

Villalpando Canchola, E. 1988. Diversidad y zonación de moluscos de facie rocosa en Isla Roqueta, Acapulco, Guerrero. Escuela Nacional de Antropología e Historia, México, Cuicuilco, México, D.F. p. 82-95.

Vokes, H. E. y E. H. Vokes. 1983. Distribution of shallowwater marine Mollusca, Yucatan Peninsula, Mexico. Mesoamerican Ecology Institute Monograph 1. Middle American Research Institute, Tulane University, New Orleans 54:182.

Wiley, G. N., R. C. Circé y J. W. Tunnel, Jr. 1982. Mollusca of the rocky shores of east central Veracruz state, Mexico. The Nautilus 96:55-61. 